\title{
Detection of Knee Unlock During Stance by Accelerometry
}

Peter H. Veltink, Member, IEEE, and Henry M. Franken

\begin{abstract}
Detection of knee unlock is a crucial part of finite state artificial reflex control of paraplegic standing supported by functional neuromuscular stimulation (FNS). This paper investigates knee unlock detection schemes using small uniaxial accelerometers mounted on the thigh and shank. Four single and two differential accelerometer configurations were evaluated with respect to knee unlock detection. Their performances were compared to goniometer-based knee unlock detection. Two accelerometers were mounted on the thigh, one just distal to the hip and one just proximal to the knee. The other two accelerometers were mounted on the shank, one just distal to the knee and the other just proximal to the ankle. The sensitive axes of all four accelerometers were directed in the sagittal plane, perpendicular to the long axes of the body segments. The first differential configuration consisted of the two accelerometers on the thigh, the second of the accelerometer on the thigh just proximal to the knee and on the shank just distal to the knee. Detection thresholds were set such that anterior-posterior body sways with locked knees would not yield false knee unlock detections. Experiments were performed in five healthy adult subjects, performing fast and slow knee flexions and anterior-posterior body sways. The first differential accelerometer configuration and the single accelerometer just proximal to the knee gave the earliest detection of fast knee unlocks, earlier than the goniometer-based detection. The second differential accelerometer configuration gave the earliest detection of slow knee unlocks, comparable to the goniometerbased detection. The first differential accelerometer configuration did not provide a reliable detection of slow knee unlocks. The single accelerometer configurations could only detect slow knee unlocks, while rejecting whole body movements, at relatively large knee flexion.
\end{abstract}

\section{INTRODUCTION}

$\mathbf{S}^{\mathrm{s}}$ TANDING is an important task to be restored in paraplegics, because it allows grasping and handling of objects in nonadapted environments [16] and communication with other individuals face-to-face.

Standing and other mobility functions can be restored in paraplegics by means of electrical stimulation of paralyzed muscles [functional neuromuscular stimulation: (FNS)] [7], [13], by orthoses, or by a combination of both [1], [15], [17]. When stimulating skeletal muscles for prolonged times, muscle fatigue is a major problem [2], [4], [12]. It can be reduced by minimizing the duration of stimulation [2], [12]. Several methods have been described to reduce stimulation of individual muscles, in most cases, by using an intermittent stimulation scheme. Kralj et al. [12] proposed open-loop alter-

Manuscript received December 7, 1994; revised October 7, 1996.

The authors arer with the Institute for Biomedical Technology (BMTI), Faculty of Electrical Engineering, University of Twente, 7500 AE Enschede, The Netherlands.

Publisher Item Identifier S 1063-6528(96)09371-8.

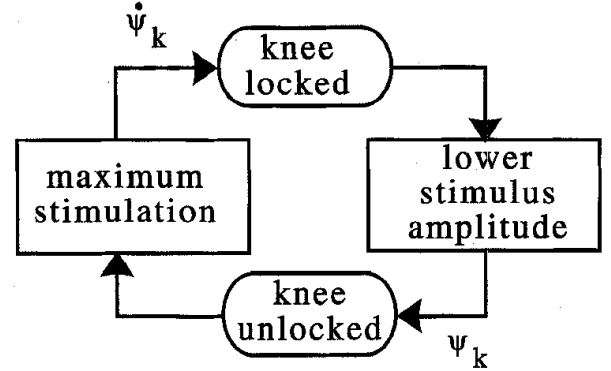

Fig. 1. Finite state control strategy for paraplegic standing with minimal quadriceps stimulation, as proposed by Mulder et al. [10], [11]. $\psi_{k}$ is the knee angle, measured by a knee goniometer; $\dot{\psi}$ is knee angular velocity, obtained by differentiating the knee angle signal.

nate stimulation of quadriceps and calf muscles under patient command (posture switching). Andrews et al. [1] proposed an ankle foot orthosis (AFO) with an additional instrumented strap above the knee. The quadriceps were stimulated when the strap was unloaded, as detected by strain gauges. In our laboratory, Mulder et al. [10], [11] proposed a finite state artificial reflex control strategy for standing (Fig. 1), which can be used with or without an AFO. When the knee was locked, quadriceps stimulation amplitude was decreased exponentially with a typical time constant of $1 \mathrm{~s}$, until knee unlock was detected by knee goniometry. After knee unlock detection, stimulation was resumed at a supramaximal level to ensure rapid knee locking. Subsequently, knee lock was detected by zero derivative of the knee angle, measured by goniometry. After knee lock detection, the goniometer offset was reset to compensate for potential goniometer slippage, and the stimulation amplitude was again gradually lowered. Moynahan and Chizeck [8] described the use of a modified pulse width and stimulus period (PWSP) control strategy, which combines pulse width and stimulus period modulation to ensure fast quadriceps response when knee unlock was detected.

In all reported closed-loop control strategies for knee control during stance [1], [8], [10], [11], the discrete detection of knee unlock is essential. A stable and well-tuned continuous controller of knee angle is practically not feasible, because of the highly nonlinear behavior of the passive characteristics of the knee at full knee extension. The alternative control of forces in the ligaments and other passive structures around the knee is not feasible, because these forces cannot be measured from the surface of the limb. Therefore, Andrews et al. [1] proposed to implement an artificial instrumented knee lock, using strain gauge instrumented straps above the 
knee, attached to a shank brace. They could detect knee unlock from the measured tension in the straps. Moynahan and Chizeck [8] and Mulder et al. [10], [11] detected knee unlock from knee kinematics, using knee goniometers. Both the artificial instrumented knee lock and the goniometer system require mechanical constructions across the knee, which may be cumbersome in daily use, and do not comply with minimal leg bracing.

Due to the bulkiness of current techniques and limitations of accuracy and reproducibility of especially the goniometer measurements [8], [10], [11], alternative knee unlock detection schemes, based on uniaxial accelerometers, were investigated. The sensors are small (the ICsensors type 3021 accelerometers used in this study have dimensions $15 \times 15 \times 5 \mathrm{~mm}^{3}$ ), and can be easily mounted on the skin using double sided tape. Furthermore, acceleration measurements may yield earlier detection of fast and sudden knee unlock than knee angle measurements, since large acceleration components in the sagittal plane, perpendicular to thigh and shank just proximal and distal to the knee will occur before the knee is substantially flexed [19].

Several authors have investigated the use of accelerometry for kinematic analysis of body movements. Kinematic analysis using accelerometers is complicated by the combined measurement of the accelerational and gravitational components. Ladin and $\mathrm{Wu}$ [6] estimated the acceleration of the center of mass of a pendulum by subtracting the gravitation component off the accelerometer signals on the basis of an independent orientation measurement using an optokinetic system. Willemsen et al. [20], [21] proposed an accelerometer sensor system consisting of two biaxial accelerometers per body segment, which enables full kinematic analysis of body movements in the sagittal plane. Willemsen's approach was based on the representation of the same kinematic vector quantity in different coordinate systems. Alternatively, by subtracting signals of equally directed uniaxial accelerometers, located on the same rigid body, it is possible to assess the angular acceleration or the square of the angular velocity of that body, depending on the orientation of the accelerometers, because the gravitational component and the acceleration of the whole body are rejected by the subtraction. This principle was used by Morris [9], who proposed a configuration of six uniaxial accelerometers, used to estimate all components of the angular acceleration of a freely moving rigid body. Padgaonkar et al. [14] proposed a configuration of nine accelerometers for the same purpose, enabling the assessment of all components of the angular accelerations without solving differential equations. Franken et al. [5] used a configuration of two accelerometers proximally and distally placed on the shank to. assess it's angular acceleration, assuming planar movements in the sagittal plane. The sensitive axes of both uniaxial accelerometers were in the sagittal plane, perpendicular to the long axis of the shank. Using this configuration Franken et al. [5] were able to estimate the knee torque generated by quadriceps stimulation outside the range of full knee extension. Veltink et al. [18] used the gravitational component of the accelerometer signal under quasistatic conditions to monitor orientations of body segments.
The objective of this study is to investigate the detection of knee unlock using uniaxial accelerometers mounted on the thigh and shank. The performances of six accelerometer systems were compared with goniometer-based detection, evaluating detection time and required knee angle detection threshold. Knee unlock detection was performed in five adult healthy subjects, who generated knee unlocks repeatably. Two modes of knee unlocks, which have been reported for FNSassisted stance in paraplegics [11], were simulated by the subjects. If standing is performed without stimulating the knee extensors, and therefore no active extension moment is acting on the knee, an external disturbance or sudden voluntary movement of the upper body may result in a fast passive knee unlock, with increasing angular acceleration as the knee flexes further. This will be called fast knee unlock. Only early detection of knee unlock and subsequent maximal knee extensor stimulation can prohibit a total collapse of the knee. Alternatively, knee unlock may occur slowly, if the electrically stimulated muscles cannot stabilize the knee at the current stimulation amplitude due to fatigue or the reduction of the stimulation amplitude, as in the protocol of Mulder et al. [10], [11]. Initially the gradual loss of knee extension torque generated by the stimulated muscles is compensated by reduced passive torque as the knee flexes out of the knee lock. The actual speed of this slow knee flexion depends on how fast the actively generated knee extension torque diminishes.

During quiet stance, small anterior-posterior movements may occur, varying the center of pressure under the foot support area without knee unlock. The knee unlock detection schemes should be designed such that they do not erroneously detect these movements.

\section{THEORY}

\section{A. Kinematic Measurements Using Uniaxial Accelerometers}

Seismic uniaxial accelerometers measure the component $a_{u}$ of an equivalent acceleration, $\vec{a}_{\text {eq }}$, in the direction of the sensitive axis of the accelerometer (Fig. 2)

$$
a_{u}=\vec{a}_{\mathrm{eq}} \cdot \vec{u}
$$

where $\vec{u}$ is the unit vector in the direction of the sensitive axis of the accelerometer.

The equivalent acceleration, $\vec{a}_{\text {eq }}$, is the sum of the acceleration of the sensor $\vec{a}$ and the equivalent gravitational acceleration $\vec{g}$ acting on the seismic mass (Fig. 2)

$$
\vec{a}_{\mathrm{eq}}=\vec{a}-\vec{g}
$$

If an accelerometer is mounted on a rigid body, the acceleration $\vec{a}$ can be expressed in terms of a body fixed coordinate system (Fig. 3)

$$
\vec{a}=\vec{a}_{o}+\vec{\omega} \times(\vec{\omega} \times \vec{r})+\vec{\alpha} \times \vec{r}
$$

where $\vec{a}_{o}$ is the acceleration of the origin of the body fixed coordinate system, $\vec{\omega}$ is the angular velocity, $\vec{\alpha}$ is the angular acceleration of the rigid body, and $\vec{r}$ is the distance from the body fixed origin to the accelerometer. 


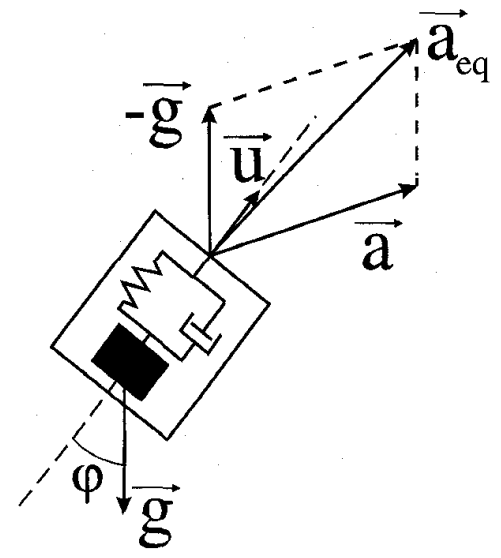

Fig. 2. Seismic uniaxial accelerometers measure the component of an equivalent acceleration $\vec{a}_{\text {eq }}$ in the direction $\vec{u}$ of the sensitive axis of the accelerometer. This equivalent acceleration $\vec{a}_{\text {eq }}$ is the sum of the acceleration of the sensor $\vec{a}$ and the equivalent gravitational acceleration $\vec{g}$ acting on the seismic mass. $\varphi$ is the angle between the sensitive axis of the accelerometer and the gravitational field.

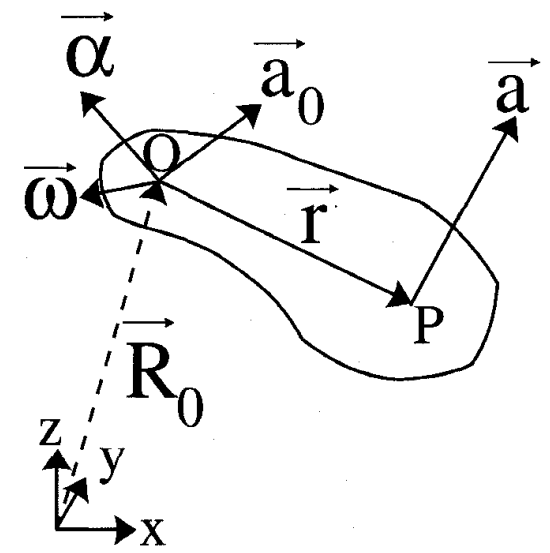

Fig. 3. The acceleration $\vec{a}$ at a point $\mathrm{P}$ of a rigid body can be expressed in terms of the acceleration $\vec{a}_{o}$ of a reference point $O$ of that body expressed in the reference coordinate system, the distance $\vec{r}$ from the reference point, and the angular acceleration $\vec{\alpha}$ and angular velocity $\vec{\omega}$ of the body.

If two uniaxial accelerometers are mounted on the same rigid body at distances $\vec{r}_{1}$ and $\vec{r}_{2}$ from the origin of this body and if their sensitive axes are in parallel, the common gravitational component and the acceleration of the body origin $\vec{a}_{o}$ are rejected in the difference signal [9]

$$
a_{u 2}-a_{u 1}=\left[\vec{\omega} \times\left(\vec{\omega} \times\left(\vec{r}_{2}-\vec{r}_{1}\right)\right)+\vec{\alpha} \times\left(\vec{r}_{2}-\vec{r}_{1}\right)\right] \cdot \vec{u}
$$

where $a_{u 2}$ and $a_{u 1}$ are the accelerometer signals at distances $\vec{r}_{1}$ and $\vec{r}_{2}$ from the body origin.

If the sensitive axes of the accelerometers are also aligned perpendicular to the vector $\vec{r}_{1}-\vec{r}_{2}$, only the angular acceleration term is left in the difference signal

$$
a_{u 2}-a_{u 1}=\left[\vec{\alpha} \times\left(\vec{r}_{2}-\vec{r}_{1}\right)\right] \cdot \vec{u} .
$$

If the angular acceleration vector $\vec{\alpha}$ is perpendicular to the plane spanned by $\vec{u}$ and $\vec{r}_{1}-\vec{r}_{2}$, the difference signal reduces to

$$
a_{u 2}-a_{u 1}=\alpha\left(r_{2}-r_{1}\right)
$$

where $\alpha, r_{1}$ and $r_{2}$ are the magnitudes of the vectors $\vec{\alpha}, \vec{r}_{1}$ and $\vec{r}_{2}$.

Equation (6) is valid for two uniaxial accelerometers, mounted on the thigh or the shank, assuming the leg is moving in the sagittal plane only [20], [21], and if the axes of the accelerometers are in parallel and directed in this plane of movement and perpendicular to the difference vector $\vec{r}_{1}-\vec{r}_{2}$. If these conditions are not met exactly, relatively small deviations from (6) will occur, since the sensitivities of the difference signal $a_{u 2}-a_{u 1}$ to small errors in the alignment of the accelerometers with respect to the plane of movement and to the direction of the difference vector $\vec{r}_{1}-\vec{r}_{2}$, are zero at the ideal alignment.

If the acceleration $\vec{a}_{0}$ of the body-fixed origin and the angular acceleration $\vec{\alpha}$ are relatively small (quasistatic), a uniaxial accelerometer measures only one component of the gravitational acceleration $\vec{g}$

$$
a_{u}=-g \cos \varphi
$$

where $\varphi$ is the angle between the sensitive axis of the accelerometer and the direction of the gravitational field (Fig. 2).

If two accelerometers are placed on different segments of the leg, the angle between the two segments can be estimated using (7), provided the quasistatic condition is satisfied. Under dynamic conditions, however, acceleration components will be present in the signal.

\section{B. Detection of Knee Unlock Using Uniaxial Accelerometers}

In this study, knee unlock detection was investigated using accelerometers on the thigh and shank, with their sensitive axes perpendicular to the long axes of the leg segments and in the sagittal plane. During slow knee unlock these sensors will mainly give inclination information, because the gravitational component is dominant [see (7)]. During fast knee unlock, large acceleration components are also to be expected [19], especially for the accelerometers which are mounted just proximally or distally to the knee. When subtracting the signals of two parallel tangential accelerometers on the thigh, the angular acceleration of the thigh is measured [see (6)], which will yield a good detection of fast knee unlock. Subtracting the signals of the accelerometers on thigh and shank, just proximally and distally to the knee, will measure inclination differences of thigh and shank, while partly rejecting the acceleration components associated with knee unlock during stance, and therefore is expected to perform comparably to a knee goniometer. Rejection of erroneous detections of anteriorposterior movements during stance is expected to be better when subtracting the signals from two accelerometers, than in the case of single accelerometers, because the responses are common to the accelerometers if they are in parallel.

\section{METHODS}

The accelerometry- and goniometer-based knee instability detection schemes were compared experimentally in five healthy subjects (four men, one woman), with no apparent motor deficiencies. Table I provides demographic information about the subjects. 
TABLE I

Subject Information. All Subjects Were Healthy Nonimpatred Adults

\begin{tabular}{llccc}
\hline Subject & Gender & $\begin{array}{c}\text { Age } \\
\text { [years] }\end{array}$ & $\begin{array}{c}\text { Body length } \\
{[\mathrm{m}]}\end{array}$ & $\begin{array}{c}\text { Body mass } \\
{[\mathrm{kg}]}\end{array}$ \\
\cline { 1 - 1 } $\mathrm{A}$ & Male & $\frac{23}{23}$ & 1.82 & 62 \\
$\mathrm{~B}$ & Male & 27 & 1.83 & 82 \\
$\mathrm{C}$ & Male & 22 & 1.83 & 75 \\
$\mathrm{D}$ & Male & 23 & 1.81 & 90 \\
$\mathrm{E}$ & Female & 23 & 1.68 & 70 \\
\hline
\end{tabular}

\section{A. Experimental Setup}

Two rigid aluminum strips (290 $\mathrm{mm}$ in length) were mounted on the anterior surfaces of the thigh and shank of the right leg using elastic straps. Two piezoresistive uniaxial accelerometers (ICsensors ${ }^{\mathrm{TM}}$ type $3021,5 \mathrm{~g}$ ) were mounted on the ends of each strip with their sensitive axes perpendicular to the long axes of the strips and in the sagittal plane. The distance between the centers of the accelerometers on each strip was $260 \mathrm{~mm}$. The strips ensured that the axes of the accelerometers on the same leg segment remained parallel, which cannot be easily achieved when the accelerometers are mounted directly on the leg. Photographs of the accelerometer mounting on the leg are shown in Fig. 4. A schematic diagram of the sensor system with numbering of the accelerometers is given in Fig. 5. The average distance from the estimated center of rotation of the knee to the center of the distal thigh accelerometer was $78 \mathrm{~mm}$, and to the center of the proximal shank accelerometer $81 \mathrm{~mm}$. The knee angle was measured by a Penny and Giles ${ }^{\mathrm{TM}}$ strain gauge based flexible goniometer, mounted laterally over the knee joint. The terminals of the goniometer were attached to the skin using double sided tape. It appeared that the reproducibility and the accuracy of the goniometer measurements were limited to approximately $2^{\circ}$. The resolution was approximately $0.5^{\circ}$.

Unlock detection was assessed for the four single accelerometers and the two differential configurations, with respect to the goniometer-based knee unlock detection. The first differential configuration, called 2-1, consisted of the two thigh accelerometers (\#1 and \#2 in Fig. 5). The second differential configuration, called 2-3, consisted of the distal thigh accelerometer ( $\# 2)$, and the proximal shank accelerometer (\#3). For both differential configurations, the difference of the accelerometer signals ( $a_{u 2}-a_{u 1}$ and $a_{u 2}-a_{u 3}$, respectively) was used for detection of knee unlock.

The accelerometer and goniometer signals were sampled at $100 \mathrm{~Hz}$ by a PC-AT compatible computer, equipped for data acquisition (Analog Devices RTI-815, 12-bit). The accelerometer signals were filtered with a first order filter (cut off frequency of approximately $35 \mathrm{~Hz}$ ) prior to sampling, which resulted in an effective delay of approximately $5 \mathrm{~ms}$ for frequencies less than $25 \mathrm{~Hz}$. The accelerometers were calibrated with static $+1 \mathrm{~g}$ and $-1 \mathrm{~g}$ reference measurements.

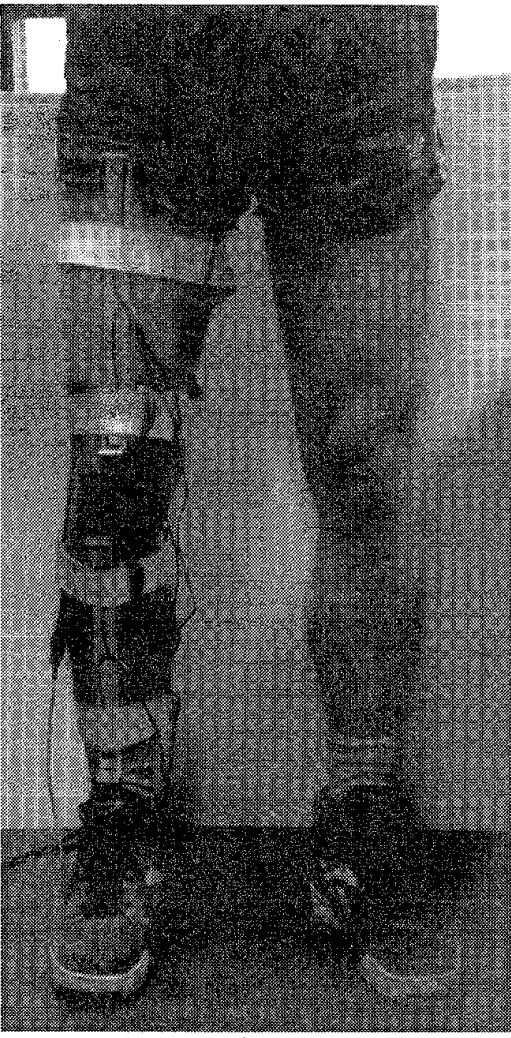

(a)

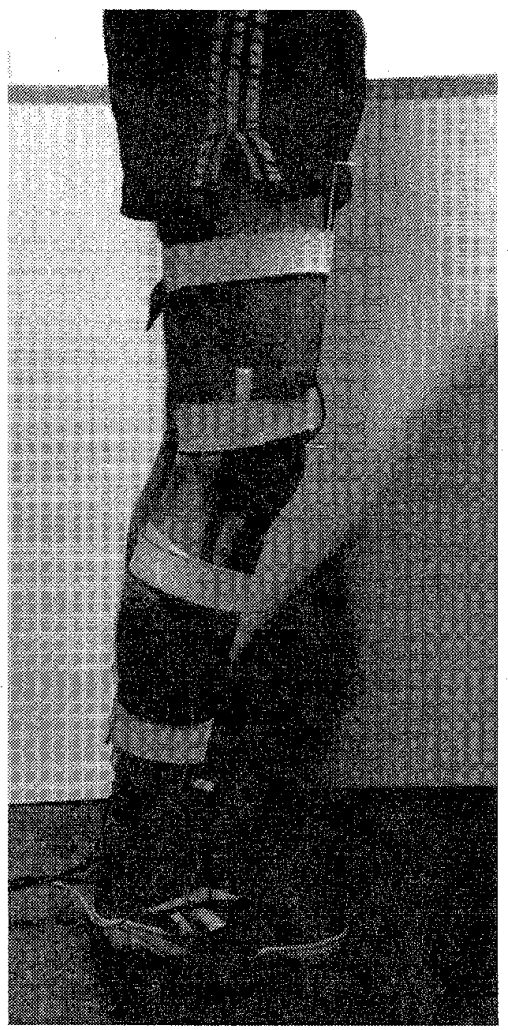

(b)

Fig. 4. Photograph of a subject flexing his legs, with the accelerometers and goniometer mounted on his right leg. (a) Front view. (b) Side view. 


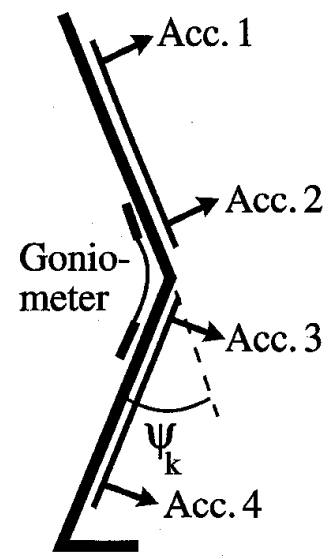

Fig. 5. Schematic diagram of the sensor system used in the experiments.

The goniometer was calibrated on the body: the offset was measured during stance with the knees fully extended. The gain was determined from an additional measurement at $90^{\circ}$ knee flexion, which was checked with a $90^{\circ}$ reference object. The estimated accuracy of the knee angle measurement after calibration is $2^{\circ}$ in the knee flexion range from 0 to $90^{\circ}$. It appeared that the sensitivity found with this calibration on the body was different from a calibration on the bench. This may be due to movements of the skin at both terminals of the goniometer or to nonideal alignment of the terminals in the sagittal plane.

\section{B. Experimental Protocol}

The five subjects performed three trials: a fast knee unlock trial, a full body sway trial and a slow knee unlock trial. The order of these trials were not random, but fixed for all subjects. The trials represented the different modes of knee unlock (fast and slow), as previously presented, and a full body anterior-posterior sway during stable unsupported stance without knee unlock, which should not lead to false detections. During the fast knee unlock trials, the subjects were instructed to force both knees to unlock ten times, relaxing their muscles around the knee joint as much as possible. Subsequently, the subjects were instructed to voluntary restore full knee extension and to stand quietly for approximately $5 \mathrm{~s}$. During the full body sway trial, the subjects were instructed to move their bodies ten times slowly in the anterior and posterior directions, while remaining their standing stability and without flexing their knees and hips. For the slow knee flexion trials, the subjects were instructed to perform ten trials of slow knee flexion of approximately 1 second duration and to approximately 30 to $40^{\circ}$ of knee flexion, with subsequent restoration to full knee extension, and to stand quietly for approximately $5 \mathrm{~s}$. The subjects practiced the instructed movements several times before actual measurement to avoid training effects.

\section{Off-Line Data Analysis}

Detection of knee unlock was evaluated off-line for the knee goniometer, the four single accelerometer configurations, and both differential configurations (2-1 and 2-3) in the following manner. First, critical threshold values were determined for each subject, such that no knee instability was detected for the full body sway trials and for the quiet stance periods between the knee flexions/extensions in the knee unlock trials. Subsequently, the detection of all knee unlocks was evaluated and timed for all accelerometer and goniometer configurations.

The critical thresholds for rejection of false knee unlock detections due to the full body sways were expressed in the equivalent variations of thigh, shank and knee angles, assuming quasistatic movements [see (7)]. This could not be done for configuration $2-1$, because the difference signal for this configuration contains no inclination component.

The detection time differences between the goniometerbased and accelerometer-based unlock detection methods were evaluated. A one way analysis of variance (ANOVA) test was performed to test the significance of the average detection time differences between the six accelerometer detection schemes. If significant differences were found, a Scheffé test [3] was performed to investigate these differences. Statistical tests were performed using the statistical software package SPSS ${ }^{\mathrm{TM}}$.

\section{RESULTS}

Fig. 6 displays typical recordings of the goniometer signals, the single accelerometer signals $\left(a_{u 1}, a_{u 2}, a_{u 3}\right.$, and $\left.a_{u 4}\right)$ and the two differential accelerometer signals $\left(a_{u 2}-a_{u 1}\right.$ and $\left.a_{u 2}-a_{u 3}\right)$. The detection thresholds are indicated. Fig. 7 illustrates the average values and standard deviations of the detection timing differences with respect to goniometer-based detection.

The accelerometer configuration 2-1 did not provide reliable detection of slow knee unlock, because the angular accelerations of the thigh were relatively small. However, 49 of 50 knee unlocks were correctly identified, although the detection timing varied considerably, as illustrated by the relatively large standard deviation for this configuration in Fig. 7(b). As indicated in Fig. 6(a) and (b), the detection thresholds for $a_{u 3}$ and $a_{u 4}$ were set below the resting value of the signal to enable the detection of slow knee unlock by the changing gravitational component of the signal. Consequently, these configurations were relatively late in detecting fast knee unlocks [see also Fig. 7(a)].

Table II illustrates the relatively large angular thresholds that were required for rejection of false detections of full body sway movements with the single accelerometer configurations. The table specifies the equivalent quasistatic angle variations of thigh (single accelerometer configurations \#1 and \#2), shank (single accelerometer configurations \#3 and \#4), and knee (differential accelerometer configuration 2-3 and knee goniometer), observed during the body sway trials. The equivalent quasistatic angle ranges for the accelerometer configurations were determined using (7). This table illustrates that slow knee unlock can be detected at smaller knee angle thresholds for the goniometer and the differential accelerometer configuration 2-3 than for the single accelerometer configurations. These lower thresholds are due to the fact that, under quasistatic conditions, both the goniometer and differential accelerometer configurations evaluate the angular deviation between the thigh 


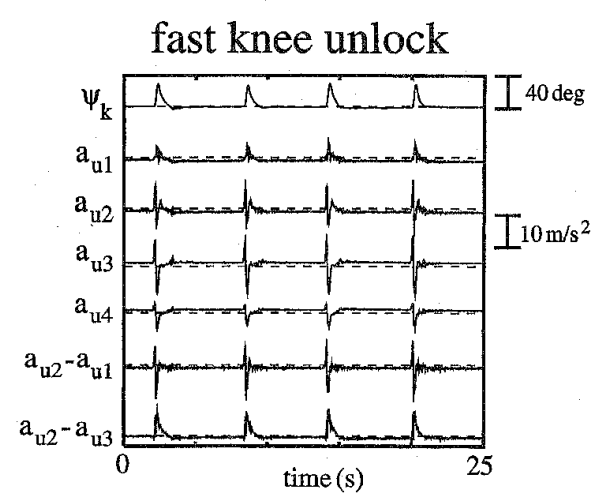

(a)

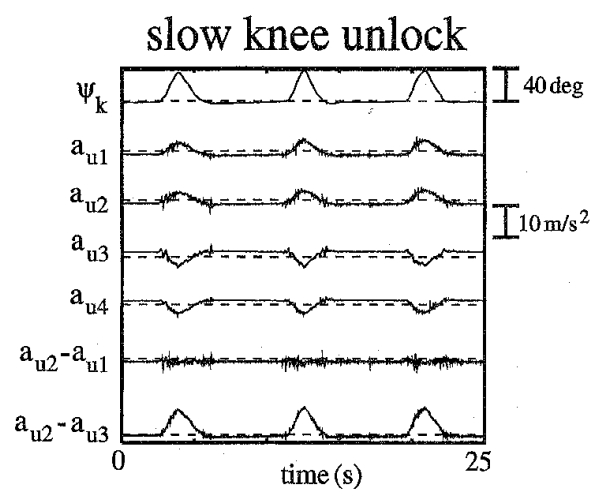

(b)

Fig. 6. Typical recordings of the goniometer signal $\psi_{k}$, the signals of the single accelerometer configurations $a_{u 1}, a_{u 2}, a_{u 3}$, and $a_{u 4}$ and of the differential accelerometer configurations $a_{u 2}-a_{u 1}$ and $a_{u 2}-a_{u 3}$ for fast (a) and slow knee unlock (b). The detection thresholds are indicated by the dashed lines.

and shank, which is equal to the knee angle. This joint angle is relatively insensitive to whole body movements. When interpreting the angle variations specified in Table II, it should be noted that a given knee unlock detection threshold for the thigh or shank angles, as measured quasistatically by the single accelerometer configurations, corresponds to a knee angle threshold, as measured by the knee goniometer and quasistatically by configuration 2-3, which is approximately twice as large. However, the required thresholds for the single accelerometer configurations are approximately half the angles indicated in the table, because the thresholds are only determined by the posterior excursion of the thigh (configurations 1 and 2), and by the anterior excursion of the shank (configurations 3 and 4). In conclusion, the angles of the thigh and shank presented in Table II give an indication of the knee angle at which knee unlock will be detected with these single accelerometer configurations.

The ANOVA test showed signficant differences in average timing of knee-unlock detections between the six accelerometer configurations for both fast and slow knee unlocks ( $\alpha=$ $0.05)$. For the fast knee unlock trials, the Scheffé test showed that configurations 2 and 2-1 gave a significantly earlier detection of knee-unlock than the other accelerometer configurations, and that configurations 1 and $2-3$ gave a significantly

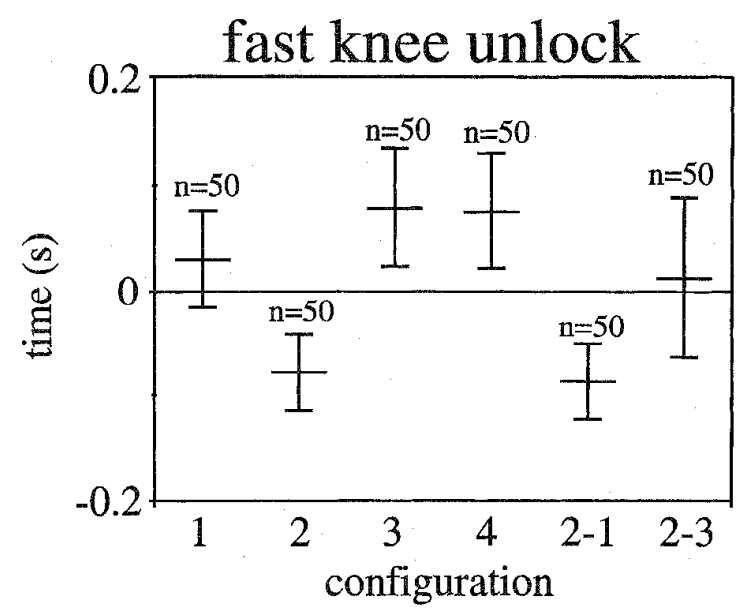

(a)

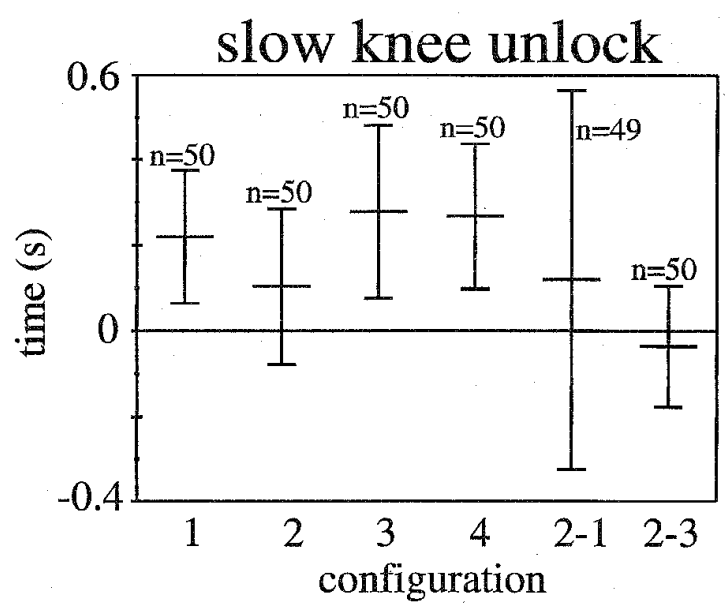

(b)

Fig. 7. Average and standard deviations of detection time differences of the accelerometer configurations with respect to goniometer-based detection for the fast knee unlock trials (a) and the slow knee unlock trials (b). Configurations $1,2,3$, and 4 are the single accelerometer configurations; configurations 2-1 and 2-3 are the differential accelerometer configurations. A positive detection time difference means that detection is later than goniometer-based detection, a negative time difference means earlier detection; $n$ is the number of correctly detected knee unlocks out of 50 .

earlier detection of knee-unlock than configurations 3 and 4 $(\alpha=0.05)$. For slow knee unlocks, configuration 2-1 was excluded from the statistical analysis of detection timing, because of the large standard deviation of the detection timing in comparison to the other accelerometer configurations. For slow knee unlock, configuration 2-3 was shown to give a significantly earlier detection of knee-unlock than the other configurations, while configuration 2 gave a significantly earlier detection than configurations 1,3 , and 4 .

\section{DISCUSSION}

Accelerometers are an alternative to knee goniometers for detection of slow and fast knee unlock during stance. However, a single accelerometer cannot reject false knee unlock detections for whole body movements without compromising adequate detection of actual knee unlock during stance. A 
TABLE II

Equivalent Quasistatic Angle Variations of Thigh (Single Accelerometer Configurations \#1 and \#2), Shank (Single Accelerometer Configurations \#3 and \#4) and Knee (Differential Accelerometer Configuration 2-3 and Knee Goniometer), ObSERved During the Body Sway Trials. The Equivalent Quasistatic Angles for the Accelerometer Configurations Were Determined Using (7). These Angles indicate the ReQuired Threshold for Rejection of False Knee Unlock Detections in the Case of Whole Body Movements

\begin{tabular}{|c|c|c|c|c|c|c|c|}
\hline & \multirow{3}{*}{$\begin{array}{l}\text { Sensor } \\
\text { Configuration }\end{array}$} & \multicolumn{2}{|c|}{ thigh } & \multicolumn{2}{|c|}{ shank } & \multicolumn{2}{|c|}{ knee } \\
\hline & & \multicolumn{2}{|c|}{ accelerometer } & \multicolumn{2}{|c|}{ accelerometer } & \multirow{2}{*}{$\begin{array}{c}\text { accel. } \\
2-3\end{array}$} & \multirow{2}{*}{$\begin{array}{l}\text { gonio- } \\
\text { meter }\end{array}$} \\
\hline & & 1 & 2 & 3 & 4 & & \\
\hline \multirow{2}{*}{. } & Average (deg) & 17.3 & 18.4 & 18.1 & 15.7 & 8.9 & 5.8 \\
\hline & Std.Dev. (deg) & 2.6 & 3.8 & 3.9 & 2.8 & 2.5 & 2.4 \\
\hline
\end{tabular}

differential accelerometer configuration, with one accelerometer proximal to and one distal to the knee (configuration 2-3) yields the most reliable detection of knee unlock for slow as well as fast knee flexions, without false detection of whole body movements. However, the single accelerometer configuration 2 and the differential accelerometer configuration 2-1 enable significant earlier detection of fast knee unlock. Therefore, the differential accelerometer configuration 2-3 is the most suitable knee unlock detection system based on accelerometry for the finite state artificial reflex control strategy for FNS-supported standing, as reported by Mulder et al. [10], [11], with comparably fast detection of knee unlock as a knee goniometer. This accelerometer configuration can be combined with a simultaneous detection of fast knee unlocks on the basis of a single thigh accelerometer, just proximal of the knee (configuration 2).

A full body sway test was used to determine the threshold for knee unlock detection. In the case of standing without arm support, this test might be too stringent, because quadriceps contraction is also necessary for safety during posterior leaning, prohibiting knee flexion, which would likely result. This implies that quadriceps should not only be activated when the knee unlocks, but also when posterior tilting of the thigh is detected. However, if balance is additionally supported with the arms this consideration does not hold in general.

The experimental trials were performed in a fixed order. The full body sway trials were performed between fast and slow knee-unlock trials to minimize the possible influence of goniometer offset shift on the knee-unlock detection. The thresholds were determined off-line from the full body sway trials and applied to the fast and slow knee-unlock trials, which came immediately before and after the full body sway trial. Fatigue is not expected to be of importance in the experiments, since the experiments were not fatiguing for the participating healthy subjects. The influence of learning was reduced, by first have the subject practice the movements before the actual measurements were performed.

The current study involved a limited number of nonimpaired healthy subjects. Although five subjects can yield significant results on an inter-subject level, the study cannot be interpreted as representative for other subject categories, such as paraplegic patients standing using FNS. However, this study gives general insight in the advantages and disadvantages of possible accelerometer configurations, which validate the predictions set out on the basis of the initial theoretical analysis. The applicability of accelerometer-based knee-unlock detection in paraplegic subjects standing with FNS is still to be tested experimentally.

If knee unlock is to be detected while wearing an AFO [1], the ankle angle, and thus the angle of the shank, can be assumed to be relatively constant. In such a case, a single accelerometer mounted on the distal thigh, might yield detection results comparable to the differential configuration which has an additional accelerometer just distal to the knee.

The current study shows that comparable or improved detection of knee unlock can be achieved using accelerometer configurations. However, in comparison with goniometers, accelerometer signals have a higher bandwidth, which may result in more frequent false detections of knee unlock under circumstances that are less controlled than in this study. This noise sensitivity may be reduced by more stringent low pass filtering of the accelerometer signals. As signal components below $10 \mathrm{~Hz}$ are only relevant for the detection of the knee unlock, the bandwidth of such a filter could be set at this frequency, while the order of the filter should be sufficiently high (e.g., fourth-order Butterworth filter) to avoid effective time shifts of signal components. The alternative use of a less critical threshold value is not desirable because it has proportional effects on detection timing and effective threshold knee angle.

\section{ACKNOWLEDGMENT}

The authors would like to thank A. W. Verboon and the subjects for their contribution to the experiments and C. Baten for his advice on statistics.

\section{REFERENCES}

[1] B. J. Andrews, R. W. Barnett, G. F. Phillips, and C. A. Kirkwood, "Rule-based control of a hybrid FES orthosis for assisting paraplegic locomotion," Automedica, vol. 11, pp. 175-199.

[2] H. B. K. Boom, A. J. Mulder, and P. H. Veltink, "Fatigue during functional neuromuscular stimulation," in Progress in Brain Research. J. H. J. Album, D. J. Allum-Mecklenburg, F. P. Harris, and R. Probst, Eds. New York: Elsevier, 1993, vol. 97, ch. 36, pp. 409-418.

[3] J. L. Devore, "Probability and statistics for engineering and the sciences," Brooks/Cole, Monterey, CA, 1987. 
[4] H. M. Franken, P. H. Veltink, M. Fidder, and H. B. K. Boom, "Fatigue of intermittently stimulated paralyzed human quadriceps during imposed cyclical lower leg movements," J. Electromyograph. Kinesiology, vol. 3, pp. 3-12, 1993.

[5] H. M. Franken, P. H. Veltink, R. Tijsmans, H. Nijmeijer, and H. B. K. Boom, "Identification of quadriceps-shank dynamics using randomized interpulse interval stimulation," IEEE Trans. Rehab. Eng., vol. 3, pp. 182-192, 1995.

[6] Z. Ladin and G. Wu, "Combining position and acceleration measurements for joint force estimation," J. Biomechan., vol. 24, pp. 1173-1187, 1991.

[7] E. B. Marsolais and R. Kobetic, "Functional walking in paralyzed patients by means of electrical stimulation," Clin. Orthopaed, vol. 175, pp. $30-36,1983$

[8] M. Moynahan and H. J. Chizeck, "Characterization of paraplegic disturbance response during FNS standing," IEEE Trans. Rehab. Eng., vol. 1 , pp. 43-48, 1993.

[9] J. R. W. Morris, "Accelerometery-A technique for the measurement of human body movements," J. Biomechan., pp. 729-736, 1973.

[10] A. J. Mulder, H. B. K. Boom, H. J. Hermens, and G. Zilvold, "Artificialreflex stimulation for FES-induced standing with minimum quadriceps force," Med. Biol. Eng. Comput., vol. 28, pp. 483-488, 1990.

[11] A. J. Mulder, P. H. Veltink, H. B. K. Boom, and G. Zilvold, "Low level finite state control of knee joint in paraplegic standing," J. Biomed. Eng., vol. 14, pp. 3-8, 1992.

[12] A. Kralj, T. Bajd, R. Turk, and H. Benko, "Posture switching for prolonging functional electrical stimulation standing in parplegic patients," Paraplegica, vol. 24, pp. 221-230.

[13] A. Kralj and T. Bajd, Functional Electrical Stimulation: Standing and Walking After Spinal Cord Injury. New York: CRC Press, 1989.

[14] A. J. Padgaonkar, K. W. Krieger, and A. I. King, "Measurement of angular acceleration of a rigid body using linear accelerometers," $J$. Appl. Mechan., vol. 42, pp. 552-556, 1975.

[15] M. Solomonow, R. Baratta, S. Hirokawa, N. Rightor, W. Walker, P. Beaudette, H. Shoji, and R. D'Ambrosia, "The RGO generation II: Muscle stimulation powered orthosis as a paractical walking system for thoracic paraplegics," Orthoped, vol. 12, pp. 1309-1315, 1989.

[16] R. J. Triolo, B. W. B. Reiley, W. Freedman, and R. R. Betz, "Development and standardization of a clinical evaluation of standing function: The functional standing test," IEEE Trans. Rehab. Eng., vol. 1, pp. 18-25, 1993.
[17] P. H. Veltink, B. F. J. M. Koopman, H. J. Hermens, H. M. Franken, G. Baardman, Th. M. M. Cloostermans, J. A. van Alsté, G. Zilvold, H. J. Grootenboer, and H. B. K. Boom, "Restoration of gait in paraplegics by functional electrical stimulation and orthoses (Hybrid Systems)," in Studies in Health Technology and Informatics, Volume 9: Rehabilitation Technology Strategies for the Europen Union, E. Ballabio, I. PlacenciaPorrero, and R. Puig de la Bellacasa, Eds. IOS press, 1993, pp. 60-64.

[18] P. H. Veltink, H. B. J. Bussmann, F. Koelma, H. M. Franken, W. I. J. Martens, and R. C. van Lummel, "The feasibility of posture and movement detection by accelerometry," in Proc. 15th Annu. Int. Conf. IEEE-EMBS, San Diego, CA, Oct. 1993, pp. 1230-1231.

[19] P. H. Veltink, H. M. Franken, A. W. Verboon, and H. B. K. Boom "Detection of knee instability using accelerometers-Experimental test and potential use in the control of fes-assisted paraplegic standing," in Proc. 15th Annu. Int. Conf. IEEE-EMBS, San Diego, CA, Oct. 1993, pp. 1232-1233.

[20] A. Th. M. Willemsen, J. A. van Alsté, and H. B. K. Boom, "Real-time gait assessment utilizing a new way of accelerometry," J. Biomechan., vol. 23 , pp. 859-863, 1990.

[21] A. Th. M. Willemsen, C. Frigo, and H. B. K. Boom, "Lower extremity angle measurement with accelerometers - Error and sensitivity analysis," IEEE Trans. Biomed. Eng., vol. 38, pp. 1186-1193, 1991.

Peter H. Veltink (S'85-M' 88 ), for a photograph and biography, see this issue, p. 384 .

Henry M. Franken received the M.Sc. degree in electrical engineering from the University of Twente, Enschede, The Netherlands, in 1990 in the topic of Cartesian control of a six-link robot. He received the Ph.D. degree cum laude in the topic of control system design for FES-assisted paraplegic gait at the Institute of Biomedical Technology (BMTI) of the University of Twente.

He is currently a Member of the scientific staff of the Telematics Research Center (TRC), the Netherlands, applying systems engineering to business process design. His research interests involve applications of systems engineering. 